\title{
THE STORY OF A DISCOVERY: HOW WE FOUND THE LONG-SOUGHT-AFTER HIGGS BOSON
}

I Chiara Mariotti - INFN Torino, Italy - DOI: https://doi.org/10.1051/epn/2019502

The Higgs boson has been discovered in 2012 by the ATLAS and CMS experiments at the LHC, $\mathbf{5 0}$ years after its prediction. The scientific and human adventure of this discovery will be summarized in this article, going back to the search at LEP and to the foundation of the LHC Higgs Cross Section working group.

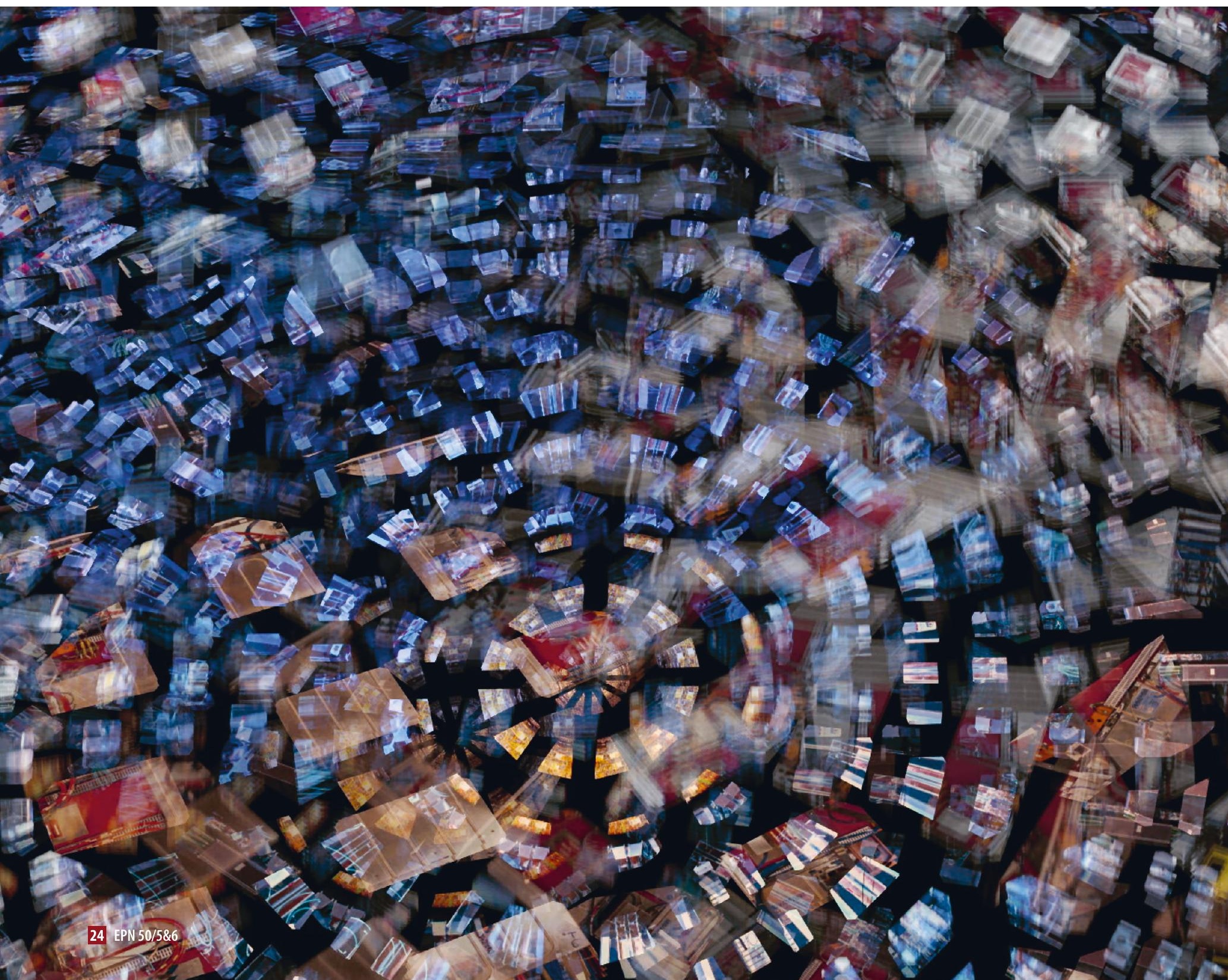




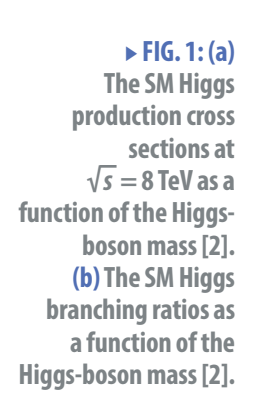

Higgs-boson mass [2].

$\mathrm{CL}$ of $\mathrm{m}_{\mathrm{H}}>114.4 \mathrm{GeV}$, with an expected limit of $115.3 \mathrm{GeV}$ [1]. The observed limit was lower than the expected since there were four events with a non-negligible probability to be Higgs boson candidates. Indeed, immediately after the end of the run, before all the final alignments and calibrations of the detectors, there were even more significant candidates that gave a probability of 0.00065 of the fluctuation to be compatible with the background only hypothesis. The initial excitement faded after the reprocessing of the data with final calibrations, when the compatibility with the background only hypothesis became 0.0024 , i.e. less than 2 sigma effect.

The LEP accelerator ended operation in December 2000 and the dismantling of the experiments and the accelerator started immediately.

In 1995 Siemens had offered to produce 32 Superconducting Radio Frequency cavities for $32 \mathrm{MCHF}$, in addition to the ones already ordered, before dismantling the production line. With those cavities LEP could have reached $\mathrm{E}_{\mathrm{cm}}=220 \mathrm{GeV}$, thus $\mathrm{m}_{\mathrm{H}}<\mathrm{E}_{\mathrm{cm}}-\mathrm{m}_{\mathrm{Z}} \sim 129\left( \pm \Gamma_{\mathrm{Z}}\right)$ $\mathrm{GeV}$. The CERN management, under pressure to get the LHC approved, decided not to increase the energy to more than $200 \mathrm{GeV}$ and leave then the future to LHC.

In reality LEP went up to $209 \mathrm{GeV}$, because the RF cavities worked very well, and with a very ingenuous trick, that was "increasing" the radius of the collider, by moving the orbit of the electrons and positrons to the maximal possible external side of the beam pipe.

\footnotetext{
${ }^{1}$ The luminosity is a performance parameter of the accelerator that allows to estimate how many events will be produced for a process with a given cross section. It is measured as the inverse of a cross section.
}

\section{Towards LHC}

In December 2000 LEP stopped and it was dismantled. LHC started the engineering work. The ATLAS detectors was built in situ in the experimental cavern, CMS on the surface and lowered between November 2006 and August 2008 to the underground cavern. The early commissioning was performed using cosmic rays. In September 2008 proton beams were circulated for the first time, but due to an accident, the first interactions were given only in November 2009, after 1 year of tremendous reparation work.

In the years 2010-2012 LHC delivered p-p collisions at energies of 7 and $8 \mathrm{TeV}$.

\section{The LHC Higgs Cross Section working group}

The same day that LHC delivered the first proton-proton collisions to the experiments, a small group of physicists met in Torino, to give birth to the group called "LHC Higgs Cross Section working group” (LHCHXSWG).

In 2008 Giampiero Passarino had the idea of the group for the first time, emphasizing the urgency, since a discovery could come sooner than expected.

In August 2009 we met at CERN with two physicists from ATLAS, and in Torino in November, the group was formed, and the program was discussed. In January 2010 the experiments formally recognized and endorsed the activities of the group. The first Yellow Report (a highly reputed CERN Report series traditionally published with a distinctive Yellow cover) was published in February 2011 [2]. This meant that since day zero the Higgs analyses from ATLAS and CMS have been using the LHCHXSWG prescriptions and results.

It was not all roses and flowers, of course! The first hard moment was between Christmas 2010 and New Year's eve 2011 when we had to submit the first Yellow Report. Some of us will hardly forget that winter break! Another hard moment was at the end of 2014, that we can summarize with this sentence: "when the battle is won the generals are coming". Thus some drastic change in the management was done.

We have published four yellow reports, that have been extensively used and cited. The group increased from $\sim 50$ authors to more than 350 . The two plots of Figure 1 were the most used ones in all the Higgs boson analyses: the cross section for the Higgs boson productions at LHC and the branching ratio of its decays.

\section{The first years of LHC data taking}

The first years of data taking were a successive series of emotions; adrenaline was flowing without interruption. We (CMS) had a first spectacular four-muons events in September 2010 with a four-muons invariant mass $\mathrm{m}(4 \mathrm{l})=201.7 \mathrm{GeV}$, after having collected $35 \mathrm{pb}^{-1}$ of luminosity ${ }^{1}$ at $7 \mathrm{TeV}$. 
For the EPS conference in Grenoble, in July 2011 we went into panic mode because of two intriguing four leptons events at $\mathrm{m}(4 \mathrm{l}) \sim 145 \mathrm{GeV}$. We had to scrutinise the data, the Monte Carlo generators, going into the details of the diagrams, interferences, parameter setting etc. The collaboration put us under a tight review, but we finally were approved and (surprise!) at the EPS2011 ATLAS presented a similar intriguing event with the same mass.

The 13 of December 2011, ATLAS and CMS presented preliminary analyses of the Higgs boson search with $4.5 \mathrm{fb}^{-1}$ of luminosity at $7 \mathrm{TeV}$. Both experiments presented an exclusion at 95\% CL for Higgs of masses larger than $\sim 130 \mathrm{GeV}$ and lighter than $\sim 115 \mathrm{GeV}$. An excess of about 3 sigma was observed by both collaborations between $\sim 115$ and $\sim 130 \mathrm{GeV}$.

In parallel, precision electroweak measurements from LEP and Tevatron suggested that the Higgs should be light, i.e. less than $130 \mathrm{GeV}$.

Both experiments decided not to look at the data in 2012 until the estimated statistics to discover an eventual Higgs boson of mass around 115-130 GeV were collected.

\section{June and July 2012}

And here comes the $14^{\text {th }}$ of June 2012, in the CMS experiment. The analysis is ready, the background is under control, the statistics is enough to observe a Higgs if its mass is $115-130 \mathrm{GeV}$. It is 19:00 in a room of Building 40 at CERN and with few people connected in video conference. We run the analysis program for the first time of that year on the data, and we project the result on the screen. And there it is: a beautiful peak!

We were seeing a new particle! Maybe the Higgs boson, so much sought after. From that moment, it was a crescendo of emotions! I remember not having slept four days in a row because of too much adrenaline.

The next day, in the afternoon of the $15^{\text {th }}$ of June, all the collaboration was reunited. Around the world, hundreds of physicists working in the CMS experiment connected via videoconference to a room at CERN, where the different groups showed the results. Two channels, $\mathrm{H} \rightarrow \mathrm{ZZ} \rightarrow 4$ leptons and $\mathrm{H} \rightarrow 2$ photons, showed a very nice peak. The other less sensitive channels, however, gave consistent results. Since the results were only to be released on the $4^{\text {th }}$ of July, we had to keep the maximum of confidentiality until then. The most difficult challenge was not smiling when going around CERN!

The $3^{\text {rd }}$ of July people started to camp outside the CERN Auditorium to be able to enter first thing the following morning. On the $4^{\text {th }}$ of July, the Auditorium at CERN was packed; all the former directors of CERN, Englert, Higgs, Hagen, and Guralnik were present. The first to speak was the spokesperson of the CMS experiment. The emotion was so great that we could not breathe and when he unveiled the transparency that showed the coveted 5 sigma the applause was long and loud. And then it was ATLAS, that showed a 5 sigma peak as well, at the same mass value. In Figure 2 there are the plots of two channels from the experiments [3,4].

At the end of 2012, after having collected almost $20 \mathrm{fb}^{-1}$ at $8 \mathrm{TeV}$, each channel had a peak that could claim a discovery by itself. With the four-leptons final state we could measure the spin and parity to be consistent at 95\% CL with the one of the SM Higgs boson. The beautiful peak that we saw for the first time on the $14^{\text {th }}$ of June, was indeed the Higgs boson.

As Paul Dirac said: "The beauty of an equation is more important than its correctness, in the sense that if an equation is beautiful, sooner or later it will be demonstrated to be correct".
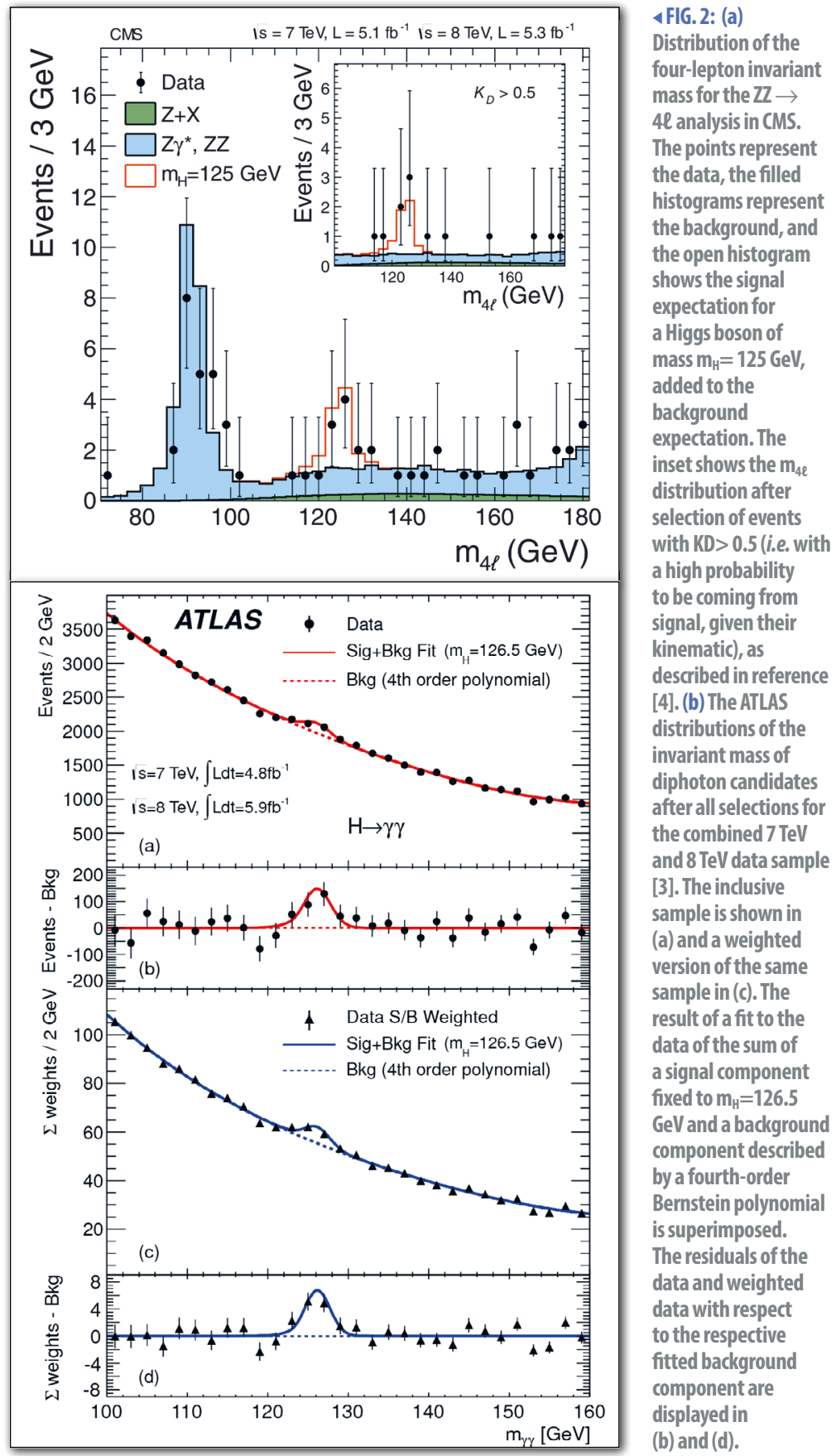

4 FIG. 2: (a) Distribution of the four-lepton invariant mass for the $\mathbf{Z Z} \rightarrow$ $4 \ell$ analysis in CMS. The points represent the data, the filled histograms represent the background, and the open histogram shows the signal expectation for a Higgs boson of mass $m_{H}=125 \mathrm{GeV}$, added to the background expectation. The inset shows the $\mathrm{m}_{4 l}$ distribution after selection of events with KD> 0.5 (i.e. with a high probability to be coming from signal, given their (a) [4]. (b) The ATLAS diphoton candidate after all selections for

[3]. The inclusive data of the sum of a signal component fixed to $\mathrm{m}_{\mathrm{H}}=126.5$ by a fourth-order Bernstein polynomial fitted background displayed in (b) and (d). version of the same 
In 2013 Prof Englert and Prof Higgs got the Nobel Prize in physics "for the theoretical discovery of a mechanism that contributes to our understanding of the origin of mass of subatomic particles, and which recently was confirmed through the discovery of the predicted fundamental particle, by the ATLAS and CMS experiment at CERN's Large Hadron Collider".

\section{Another break-through: the Higgs boson width}

In 2013, following the work of Kauer, Passarino and Caola, Melnikov, we constrained the Higgs width, $\Gamma_{\mathrm{H}}$, to few tens of $\mathrm{MeV}$ using off-shell Higgs boson production [5]. An improvement of a factor $\sim 200$, with respect to the on-shell reconstruction, dominated by the experimental resolution limiting the measurement to few $\mathrm{GeV}$.

It was an hard effort from the theoretical and experimental point of view, since we had to change all our analysis methods and Monte Carlo predictions. Thanks to many theoreticians involved in the LHCHXSWG we managed to present the first result in Moriond 2014.

The latest results with the statistics from Run 1 and half of Run 2, constrained the Higgs width to 3.2 $2_{-2.2}^{+2.8} \mathrm{MeV}$.

The Higgs boson mass of the combined ATLAS and CMS Run1 data is $m_{\mathrm{H}}=125.09 \pm 0.24 \mathrm{GeV}$, with the statistical uncertainty of $0.21 \mathrm{GeV}$ dominating over the systematic one of $0.11 \mathrm{GeV}$.

With the data collected in Run 1 and Run 2 many measurements have been possible: the Higgs boson coupling to the electroweak bosons and to all elementary fermions, differential cross sections and fiducial cross sections.

Extrapolating to the end of LHC, i.e. with $3 \mathrm{ab}^{-1}$ of luminosity per experiment, most of the couplings will be known with at $2-4 \%$ precision, dominated by theory uncertainties.

\section{Changing paradigm}

As early as July $4^{\text {th }}$, it was clear to us physicists that our world would not be the same anymore. We now know that the Higgs boson exists and is not just an elegant and fascinating theory.

Before the discovery in 2012 the hypothesis was the SM and the unknown was the mass of the Higgs boson. Therefore, bounds on $\mathrm{m}_{\mathrm{H}}$ were derived through a comparison with high precision data (LEP, Tevatron, LHC...).

After the discovery at LHC, given that the SM is fully specified, the possible unknowns are explored through deviations from the SM. Thus, schemas to define SM deviations are necessary. Two different approaches have been proposed, the $\kappa$-framework and the SMEFT (SM effective field theory) (see Yellow Reports [6,7] of the LHCHXSWG). The $\kappa$-framework is a procedure used at leading order (LO).
It is valid only if $\kappa \sim 1$, where $\kappa$ is a multiplicative factor to the SM cross section. Thus, it can just give indications if there are deviations from the SM. The SMEFT is a methodology to study possible new physics effects from massive particles that are not directly detectable. The Lagrangian of the SM is extended by introducing additional dimension-6 (or 8) operators. The underlying assumption of an effective quantum field theory is that the scale of new physics $\Lambda$ is large compared to the experimentally-accessible energies.

Moreover, we must continue to search for new particles to understand the "nature" of the newly discovered Higgs boson and try to understand if the SM is a fundamental theory. From the discovery of the neutral weak current in 1973, up to the Higgs discovery in 2012, no flaw has been detected in the SM. Is the SM just an effective field theory?

There are many open questions and many unsatisfactory explanations, and we must increase the precision of the measurements and of the predictions, especially wherever there is a little disagreement with data.

Shall we instead propose something new, completely different?

\section{Towards a new world}

We have built huge and sophisticated accelerator and detectors, "the cathedrals of science", to find an elementary particle that explain why elementary particles have mass.

The discovery of the Higgs boson is invaluable. It is a great success of a community of thousands of physicists: it is the result of a large group, where each of us has given their personal contribution.

\section{About the Author}

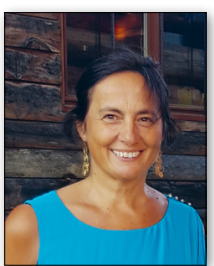

Chiara Mariotti is an elementary particles physicist of INFN-Torino. Her research activity has been focussing on data analysis in the experiments at the LEP and LHC colliders. She was one of the main actors for the Higgs boson discovery. She was awarded with the Emmy Noether Distinction for women in physics in 2018.

\section{References:}

A complete list of theoretical references can be found inside the following papers:

[1] LEP collaborations, Phys.Lett. B 565, 61 (2003)

[2] LHCHXSWG, CERN-2011-002

[3] ATLAS Collaboration Phys.Lett. B 716, 1 (2012)

[4] CMS Collaboration Phys.Lett. B716, 30 (2012)

[5] CMS Collaboration, Phys.Lett. B736, 64 (2014)

[6] LHCHXSWG, CERN-2013-004

[7] LHCHXSWG, CERN-2017-002 